See discussions, stats, and author profiles for this publication at: https://www.researchgate.net/publication/335798050

\title{
Laboratory X-ray tomography for metal additive manufacturing: round robin test
}

Article $\cdot$ September 2019

DOI: 10.1016/j.addma.2019.100837

CITATIONS

0

22 authors, including:

Anton Du Plessis

Stellenbosch University

188 PUBLICATIONS 825 CITATIONS

SEE PROFILE

F. Philip Sperling

Volume Graphics GmbH

7 PUBLICATIONS 27 CITATIONS

SEE PROFILE

Some of the authors of this publication are also working on these related projects:

Project Design of advanced materials using selective laser melting View project

Project Design of Experiments in Manufacturing View project
READS

103

Stephan Gerhard Le Roux

Stellenbosch University

49 PUBLICATIONS 173 CITATIONS

SEE PROFILE

André Beerlink

YXLON International GmbH

29 PUBLICATIONS 347 CITATIONS

SEE PROFILE 


\section{Journal Pre-proof}

Laboratory X-ray tomography for metal additive manufacturing: round robin test

Anton du Plessis, Stephan G. le Roux, Jess Waller, Philip Sperling, Nils Achilles, Andre Beerlink, Jean-François Métayer, Mirko Sinico, Gabriel Probst, Wim Dewulf, Florian Bittner, Hans-Josef Endres, Marian Willner, Ágota Drégelyi-Kiss, Tomas Zikmund, Jakub Laznovsky, Jozef Kaiser, Pascal Pinter, Stefan Dietrich, Elena Lopez, Oliver Fitzek, Porebski Konrad

PII: S2214-8604(19)30735-3

DOI: https://doi.org/10.1016/j.addma.2019.100837

Reference: ADDMA 100837

To appear in:

Received Date: $\quad 10$ June 2019

Revised Date: $\quad 23$ July 2019

Accepted Date: $\quad 21$ August 2019

Please cite this article as: Plessis Ad, le Roux SG, Waller J, Sperling P, Achilles N, Beerlink A, Métayer J-François, Sinico M, Probst G, Dewulf W, Bittner F, Endres H-Josef, Willner M, Drégelyi-Kiss Á, Zikmund T, Laznovsky J, Kaiser J, Pinter P, Dietrich S, Lopez E, Fitzek O, Konrad P, Laboratory X-ray tomography for metal additive manufacturing: round robin test, Additive Manufacturing (2019), doi: https://doi.org/10.1016/j.addma.2019.100837 
This is a PDF file of an article that has undergone enhancements after acceptance, such as the addition of a cover page and metadata, and formatting for readability, but it is not yet the definitive version of record. This version will undergo additional copyediting, typesetting and review before it is published in its final form, but we are providing this version to give early visibility of the article. Please note that, during the production process, errors may be discovered which could affect the content, and all legal disclaimers that apply to the journal pertain.

(C) 2019 Published by Elsevier. 


\section{Laboratory X-ray tomography for metal additive manufacturing: round robin test}

Anton du Plessis ${ }^{a,}{ }^{*}$, Stephan G. le Roux ${ }^{b}$, Jess Waller ${ }^{c}$, Philip Sperling ${ }^{d}$, Nils Achilles ${ }^{e}$, Andre Beerlink ${ }^{e}$, JeanFrançois Métayer ${ }^{d}$, Mirko Sinico ${ }^{f}$, Gabriel Probst ${ }^{f}$, Wim Dewulf ${ }^{f}$, Florian Bittner ${ }^{g}$, Hans-Josef Endres ${ }^{g}$, Marian Willner $^{h}$, Ágota Drégelyi-Kiss ${ }^{i}$, Tomas Zikmund ${ }^{j}$, Jakub Laznovsky ${ }^{j}$, Jozef Kaiser ${ }^{j}$, Pascal Pinter ${ }^{k}$, Stefan Dietrich k, Elena Lopez ', Oliver Fitzek', Porebski Konrad ${ }^{m}$
a. Physics Department, Stellenbosch University, Stellenbosch 7602, South Africa
b. CT Scanner Facility, Stellenbosch University, Stellenbosch 7602, South Africa
c. HX5 Teammate, NASA-JSC White Sands Test Facility, Las Cruces, NM, United States
d. Volume Graphics GmbH, Speyerer Str. 4-6, 69115 Heidelberg, Germany
e. YXLON International GmbH, a company of the COMET Group, Essener Bogen 15, D-22419 Hamburg, Germany
f. Department of Mechanical Engineering, KU Leuven, 3001 Leuven, Belgium
g. Fraunhofer Institute for Wood Research, Wilhelm-Klauditz-Institut WKI, Heisterbergallee 10A, 30453 Hanover, Germany
h. MITOS GmbH, Lichtenbergstr. 8, 85748 Garching b. München, Germany
i. Banki Donat Faculty of Mechanical and Safety Engineering, Obuda University, H-1081 Nepszinhaz u. 8. Budapest, Hungary
j. CEITEC - Central European Institute of Technology, Brno University of Technology, Czech Republic
k. Karlsruher Institut für Technologie (KIT), Institut für Angewandte Materialien - Werkstoffkunde (IAM- WK), Campus Süd, Engelbert-Arnold-Straße 4, D-76131 Karlsruhe, Germany
I. Fraunhofer Institute for Materials and Beam Technologies, Dresden, Germany
m. Laboratory of Industrial Tomography, National Centre for Nuclear Research, Poland
n.

Corresponding author: anton2@sun.ac.za

This paper reports on the results of a round robin test conducted by ten X-ray micro computed tomography (micro-CT) laboratories with the same three selected titanium alloy (Ti6Al4V) laser powder bed fusion (L-PBF) test parts. These parts were a 10-mm cube, a $60-\mathrm{mm}$ long and $40-\mathrm{mm}$ high complex-shaped bracket, and a 15-mm diameter rod. Previously developed protocols for micro-CT analysis of these parts were provided to all participants, including suggested scanning parameters and image analysis steps. No further information on the samples were provided, and they were selected from a variety of parts from a previous different type of round robin study where various L-PBF 
laboratories provided identical parts for micro-CT analysis at one laboratory. In this new micro-CT round robin test which involves various micro-CT laboratories, parts from the previous work were selected such that each part had a different characteristic flaw type, and all laboratories involved in the study analyzed the same set of parts. The $10-\mathrm{mm}$ cube contained subsurface pores just under its top surface (relative to build direction), and all participants could positively identify this. The complex bracket had contour pores around its outer vertical sides, and was warped with two arms deflected towards one another. Both of these features were positively identified by all participants. The $15-\mathrm{mm}$ diameter rod had a layered stop/start flaw, which was also positively identified by all participants. Differences were found among participants for quantitative evaluations, ranging from no quantitative measurement made, to under and overestimation of the values in all analyses attempted. This round robin provides the opportunity to highlight typical causes of errors in micro-CT scanning and image analysis as applied to additively manufactured parts. Some workflow variations, sources of error and ways to increase the reproducibility of such analysis workflows are discussed. The ultimate aim of this work is to advance the efficient use of micro-CT facilities for process optimization and quality inspections for additively manufactured products. The results provide confidence in the use of laboratory micro-CT but also indicate the need for further development of standards, protocols and image analysis workflows for quantitative assessment, especially for direct and quantitative comparisons between different laboratories.

Keywords: additive manufacturing; laser powder bed fusion; X-ray tomography; microCT; nondestructive testing; seeded flaws; flaw detection

\section{Introduction}

Metal additive manufacturing (AM) has progressed significantly in the last few years, allowing production parts to be made using a variety of materials and processes, incorporating complexity that is not possible by other manufacturing methods [1-4]. These parts can either be almost fully dense or can incorporate high levels of porosity depending on their end-use application. Parts with varying degrees of design complexity can also be made, ranging from simple parts to complex, topologyoptimized parts. In the former case, no effort is made to capitalize on the advantages of AM and it is usually possible to characterize the surface and the volume of the part using existing nondestructive evaluation (NDE) procedures. Topology optimized parts, on the other hand, often lack flat or parallel surfaces, which greatly reduces NDE inspectability because the amount of inspection surface has increased, or much of the structure is detailed or hidden [5]. This requires existing NDE procedures to 
be customized or entirely new ones to be developed. The added demands placed on NDE by additive manufacturing are exacerbated by the presence of many process-specific defects in as-built, intermediately processed, or finished parts, including different types of porosity (gas porosity, keyhole porosity, lack-of-fusion porosity), inclusions, surface roughness, residual stress, cracks, or poor dimensional accuracy [6]. The aggregate type, size and distribution of these manufacturing defects can result in compromised mechanical properties, or a nonconforming part, and consequently, an increased risk of premature failure. The need for NDE-based quality control measures to prevent the occurrence of these manufacturing flaws and to detect them when they do occur is therefore essential to the success of additive manufacturing and its further adoption in critical applications such as are found in the aerospace, nuclear and medical industries. However, the typical defects in additively manufactured parts are very small and can adopt irregular or planar geometries, or are located in difficult to inspect areas, and are, therefore, more challenging to detect than defects found in cast, wrought or forged parts. Furthermore, the effects of these defects are not yet sufficiently understood, for example, with regard to the effect on the mechanical fatigue behavior of additively manufactured materials and parts $[7,8]$. Therefore, better quantitative NDE methods are needed to accelerate the adoption of additive manufacturing for primary load-bearing structures and system critical components. X-ray computed tomography (micro-CT) has emerged as an ideal solution to characterize and classify the process-induced porosity and is now accepted as the leading method to analyze additively manufactured components non-destructively $[9,10]$. A recent comprehensive review paper [10] highlights the capability of micro-CT to not only inspect final parts, but also to inspect small coupon 'witness' samples, whose defect state is indicative of the process conditions and mirrors the defect state in the production part. The use of witness coupons allows processes to be optimized so that the occurrence of defects is minimized by judicious variation of power, laser speed, hatch spacing, layer thickness, etc. prior to building the final production part.

Although the capabilities of micro-CT are now starting to be appreciated more widely in the AM community, there is a need for standardization of inspections for measurement of AM part porosity and dimensional metrology of AM parts, in order to improve the interpretation and ultimately the proper usage of the technique [11]. Besides generic best-practice micro-CT guidelines that are broadly applicable to any material or examination object [12], specific standards are under development such as ASTM WK47031 [13] and ISO/ASTM DTR 52905 [14] that are applicable to additively manufactured parts and products. In tandem, to enhance the accuracy of the CT measurements, research has focused on the calibration and the validation of the inspection technique through physical reference objects and phantoms, see for example [15-17].

However, there have been limited efforts at simplifying and standardizing the workflows specifically for AM inspection. This includes both scan settings as well as image analysis steps for accurate 
segmentation and porosity quantification. A recent paper shows preliminary efforts at semiautomated image analysis steps for quantification of porosity in AM parts, emphasizing the importance and need of standardized image analysis procedures [18]. One of the main limitations to standardized scanning and image analysis procedures is the fact that complex AM parts of various shapes and sizes require for each case different and customized or novel scan settings and associated image analysis steps. However, process optimization can be performed by analyzing witness coupon samples with fixed geometries - allowing fixed scanning and image analysis steps using existing procedures as a point of departure. In an effort to advance the use of microCT for process optimization, a series of protocols were proposed for the analysis of a 10-mm cube for porosity [19], density [20], surface roughness [21] and even the analysis of feedstock powders [22]. These are also available on the website protocols.io [23] for continued development and improvements, and comments are welcome there. These protocols were used in a recent round robin study whereby the same parts were produced at various laser powder bed fusion laboratories and micro-CT inspection and analysis completed with identical workflows of all parts [24]. This work highlights the fact that different forms of defects (characteristic porosity types) occur depending on the process used and these can be positively identified by micro-CT. The present round robin test follows on this work, by sending selected parts from this previous batch to various micro-CT laboratories and requesting these laboratories to follow the protocols for analysis.

\section{Materials and methods}

\subsection{Round robin test description}

A previous round robin test was conducted whereby a cube, bracket and witness rod was produced using laser powder bed fusion in Ti6Al4V at various laboratories and scanned under identical conditions at the same laboratory [24]. This work showed how a variety of different types of manufacturing errors were present in all samples, with all being relatively minor (the largest value of porosity was $0.130 \%$ among all cube samples, for example). Eight sets of samples comprising of a 10 $\mathrm{mm}$ cube, a 60-mm long bracket and a 15-mm diameter, 40-mm high cylindrical rod (see Figure 1) were fabricated with 5 different L-PBF systems and different process parameters, these are described in more detail in [24], but a brief description is given here for context. All 8 sets of samples were produced using L-PBF, in all cases using their own gas atomized Ti6Al4V powder, in all cases with optimized processes as used for production. The L-PBF laboratories that contributed samples were not disclosed but included commercial and academic facilities in both university and state-funded environments, in South Africa, Germany and the United States. One laboratory provided 2 sets of samples: with one set default and the other with a single purposefully induced layered stop/start flaw (induced by physically 
stopping the process and restarting hours later). Another laboratory provided 3 sets of samples produced with varied laser scan speeds, and correspondingly varied porosity extent. The other 3 laboratories provided one set of samples each, making up a total of 8 sets of samples comprising of cube, bracket and rod each.

For the present work, one set of three parts from this prior work was selected with cube, bracket and rod from different laboratories and processes. The prior measurements of these selected parts served as reference for comparison purposes and is denoted as Participant 1 in this work. The selection was made to purposefully include different forms of defects, without informing the micro-CT round robin participants of the selection, in order to check if any of these were incorrectly detected or missed by micro-CT round robin participants.

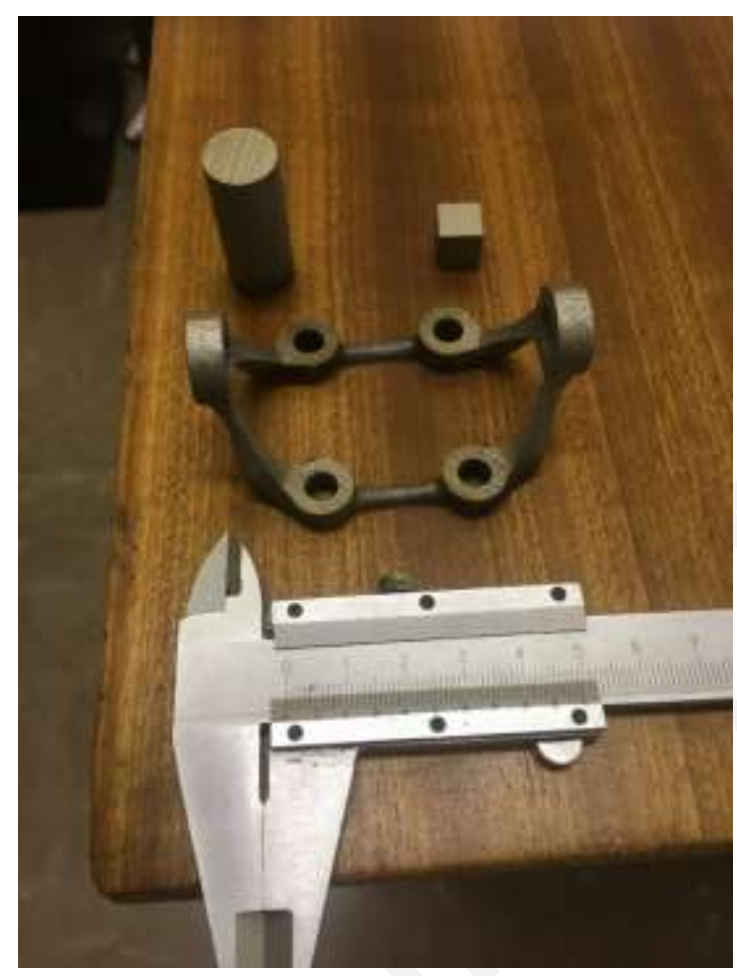

Figure 1: A typical set of samples comprising of a $10-\mathrm{mm}$ cube, $15-\mathrm{mm}$ diameter cylindrical rod and a 60-mm long bracket, from [24].

Each participant in the present round robin test was given an arbitrary participant number (1-10) which is reported here instead of laboratory names (and is not related to the order of authors). Each participant was asked a series of questions regarding the analysis - the questions are listed below, with particular reference to the previous round robin test results. All answers are given in italics - for the reference laboratory (Participant 1 ). It should be mentioned that the reference laboratory is the 
organizer of the round robin and in no way represents an ideal or more accurate measurement in any way. The aim is to compare the results from this laboratory (where the workflows originated) with those from other laboratories, focusing on the proposed workflow - with participants who have different systems, levels of experience and are given the workflow steps to follow. Results are reported in the tables exactly as provided by each participant (with different numbers of significant digits, for example). No corrections were done and results were reported directly as presented here.

1. Cube identification: Relating the scanned cube to the correct cube label $1 a, 2 a, 2 b, 2 c, 3,4$ or 5 from prior work [24]. Answer $=4$

2. Comment on the porosity distribution in the cube. Mostly spherical porosity subsurface just under top surface of cube.

3. Mean porosity value (\%) of the cube. $0.017 \%$

4. Maximum pore size in the cube (circumscribed sphere, i.e. VGSTUDIO diameter value) $.0 .19 \mathrm{~mm}$

5. Cube total material volume in $\mathrm{mm}^{3} .915 .8 \mathrm{~mm}^{3}$

6. Surface roughness $\left(\mathrm{S}_{\mathrm{a}}\right)$ value. $\mathrm{Sa}=8 \mu \mathrm{m}$, measured on side wall of cube

7. Identification of bracket: relating the scanned bracket to the correct bracket label $1 a, 1 b, 2 a, 2 b$, $2 c, 3,4$ or 5 from prior work [24]. Answer $=3$

8. Comment on the porosity distribution of bracket. Contour porosity on outer edges of bracket

9. Mean porosity for the bracket (\%). $0.056 \%$

10. Maximum pore size found in the bracket $(\mathrm{mm}) \cdot 0.75 \mathrm{~mm}$

11. For the Computer Aided Design (CAD) variance analysis -maximum deviation value: $1.12 \mathrm{~mm}$

\subsection{CT analysis procedures}

Previously developed micro-CT analysis protocols for determining the porosity [19], density [20], and surface roughness [21], and discussed elsewhere $[23,24]$ were provided to all participants, including suggested scanning parameters and image analysis steps. The scanning and image analysis steps are briefly outlined here. Scanning is performed with any typical laboratory micro-CT device, with sample loaded at an angle to minimize cone beam artefacts on flat surfaces. The cube is scanned using $200 \mathrm{kV}$ including $0.5 \mathrm{~mm}$ copper beam filtration at $15 \mu \mathrm{m}$ voxel size. The rod is scanned using similar settings at $25 \mu \mathrm{m}$, and the bracket at $45 \mu \mathrm{m}$. No image de-noising is applied and the image analysis steps are performed in Volume Graphics VGSTUDIO MAX 3.2. The steps involve selecting the surface of the cube without selecting exterior particles or internal particles or voids (in order to exactly define the edge of the material only), followed by an erosion of 2 voxels sub-surface to select all internal voxels of the cube but excluding surface defects. This new selection with its own histogram, allows a sub-voxel precision surface determination function on the edges of pores, which can be manually assessed to ensure no typical micro-CT artefacts are included. Selecting the pore spaces from this surface 
determination (termed a defect mask), allows the defect analysis function to be applied without any algorithm used. A cut-off of minimum 8 voxels is used for a pore space, to ensure no noise is detected as pore. A similar process is applied to the bracket and witness rod. Image analysis steps for surface roughness and CAD variance include the use of the "nominal-actual comparison" module of VGSTUDIO MAX and alignment is performed using best-fit registration. The results of the requested analysis from the Participant 1 (reference laboratory) are shown visually in Figure 2. Each pore diameter is reported as the diameter of the minimum circumscribed sphere which fits around it, which means this is the maximum diameter of the pore space. When multiple pores are close together and touching in the images, these will be seen as a pore cluster and the diameter of the pore cluster is reported. 


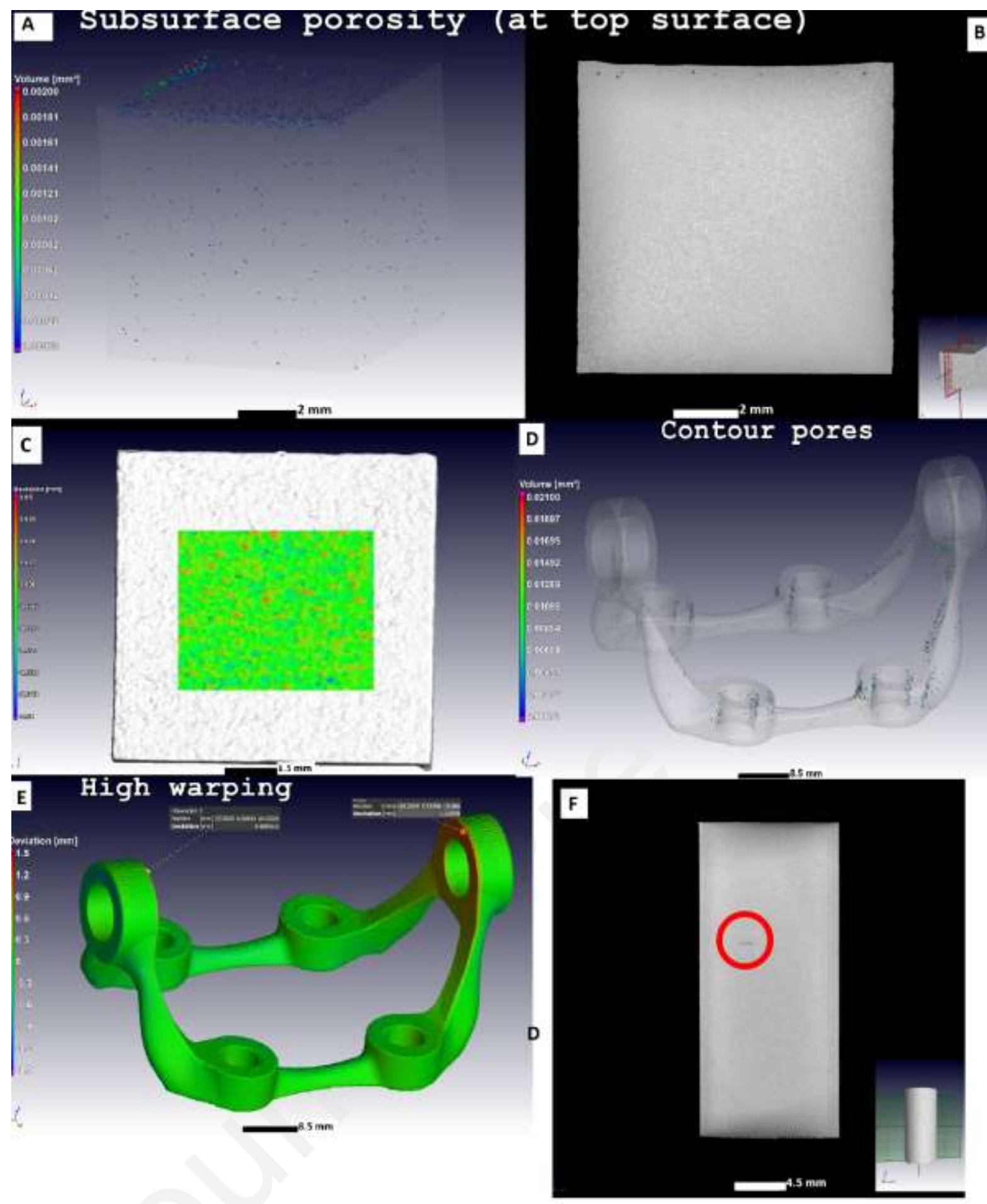

Figure 2: Reference laboratory (Participant 1 ) results showing (A) 3D porosity distribution of cube with pores at one surface only (B) 2D porosity slice image of cube (C) surface roughness colour-map (D) bracket 3D porosity analysis showing contour pores around sides of bracket (E) bracket 3D colourmap showing warping and maximum warping annotations (F) layered flaw in slice image of witness specimen (rod). Images taken from [24]. Supplementary material includes a video, a surface mesh, a coloured mesh of the pores only with colour coding according to maximum pore diameter, and full microCT slice data for the cube in (A-B). 


\section{Results \& discussion}

\subsection{Overview}

Each participant's results are discussed individually in the next section. In this section, a comparison of results is briefly outlined, with Tables 1 and 2 providing quantitative values as reported by participants. Comparing the cube analyses, it is clear that all participants could identify the correct cube (no. 4), and mention the correct porosity distribution (subsurface porosity at one side). All participants could identify pores mainly near the surfaces of the bracket and all could clearly identify the layered flaw in the rod. Besides these positive qualitative assessments, all participants encountered problems of varying degrees obtaining similar quantitative values for absolute porosity percentage and largest pore size. This is to be expected, as will be explained in more detail below.

All measurements of porosity percentage in the cube differ in the range 0.004 to $0.030 \%$, which is acceptable considering the small size and quantity of pores. Measuring porosity using micro-CT is wellestablished for large pores such as those in cast metal parts or plastic injection mouldings. However, the porosity in additive manufactured parts are typically much smaller and present in smaller quantities. With such small levels of porosity (both in pore size and number of pores), it is likely that slight differences in background image noise levels or system actual resolution might affect the degree to which small pores are detected (as many more pores are present smaller than the scan resolution). The absolute porosity value is therefore less important than the distribution of the pores. The percentage value is affected by segmentation errors, which are discussed in the next section. Suffice it to say, there were definite differences in segmentation of the images by different participants. These differences extend to the bracket analyses also, with differences noted between the participants for the maximum CAD deviation and maximum pore size detected in the bracket. All participants could, however, positively identify the contour porosity in the bracket despite some false pores detected, which are clearly due to segmentation errors and were highlighted as such by the participants involved. All participants could also identify the layered flaw in the rod. 
Table 1: Answers provided by participant related to $10-\mathrm{mm}$ cube analysis

\begin{tabular}{|c|c|c|c|c|}
\hline & $\begin{array}{l}\text { Identify } \\
\text { correct cube? }\end{array}$ & $\begin{array}{l}\text { Major } \\
\text { distribution of } \\
\text { pores }\end{array}$ & Porosity (\%) & Max pore size $(\mathrm{mm})$ \\
\hline Participant 1 & Yes & $\begin{array}{l}\text { Subsurface } \\
\text { porosity }\end{array}$ & 0.017 & 0.19 \\
\hline Participant 2 & Yes & $\begin{array}{l}\text { Subsurface } \\
\text { porosity at top } \\
\text { surface }\end{array}$ & 0.012 & 0.210 \\
\hline Participant 3 & Yes & $\begin{array}{l}\text { Subsurface } \\
\text { porosity at top } \\
\text { surface }\end{array}$ & 0.02 & 0.285 \\
\hline Participant 4 & Yes & $\begin{array}{l}\text { Mainly in edges } \\
\text { and top of cube }\end{array}$ & 0.02 & 0.54 \\
\hline Participant 5 & Yes & $\begin{array}{l}\text { Many pores close } \\
\text { to one surface }\end{array}$ & 0.03 & 0.85 \\
\hline Participant 6 & Yes & $\begin{array}{l}\text { Small subsurface } \\
\text { porosity along } \\
\text { one plane }\end{array}$ & 0.02 & 0.414 \\
\hline Participant 7 & Yes & $\begin{array}{l}\text { Subsurface } \\
\text { porosity found } \\
\text { along top surface }\end{array}$ & 0.0176 & 0.257 \\
\hline Participant 8 & Yes & No analysis & 0.0265 & 0.192 \\
\hline Participant 9 & Yes & $\begin{array}{l}\text { Pores at top } \\
\text { surface of cube }\end{array}$ & 0.0042 & 0.178 \\
\hline $\begin{array}{l}\text { Participant } \\
10\end{array}$ & Yes & $\begin{array}{l}\text { Mainly at the top } \\
\text { of the cube }\end{array}$ & 0.03 & 0.22 \\
\hline
\end{tabular}


Table 2: Answers provided relative to bracket

\begin{tabular}{|c|c|c|c|c|}
\hline & $\begin{array}{l}\text { Major } \\
\text { distribution of } \\
\text { pores }\end{array}$ & Porosity (\%) & $\begin{array}{l}\text { Max pore size } \\
(\mathrm{mm})\end{array}$ & $\begin{array}{l}\text { Warping } \\
\text { (deviation from } \\
\text { CAD) maximum } \\
(\mathrm{mm})\end{array}$ \\
\hline Participant 1 & $\begin{array}{l}\text { Contour } \\
\text { subsurface } \\
\text { pores }\end{array}$ & 0.056 & 0.75 & 1.12 \\
\hline Participant 2 & Contour pores & 0.04 & 1.58 & $>0.5$ \\
\hline Participant 3 & $\begin{array}{l}\text { Subsurface } \\
\text { pores }\end{array}$ & 0.02 & 0.48 & 0.92 \\
\hline Participant 4 & Round all holes & 0.14 & 6.46 & 1.02 \\
\hline Participant 5 & Contour pores & No analysis & No analysis & 0.85 \\
\hline Participant 6 & $\begin{array}{l}\text { Clustered } \\
\text { mainly at } \\
\text { contours }\end{array}$ & 0.02 & 0.76 & - \\
\hline Participant 7 & $\begin{array}{l}\text { Between infill } \\
\text { and contour }\end{array}$ & 0.0178 & 0.61 & 0.94 \\
\hline Participant 8 & Contour pores & 0.06 & 0.9 & 0.93 \\
\hline Participant 9 & $\begin{array}{l}\text { Close to } \\
\text { surface }\end{array}$ & 0.067 & 0.85 & 0.88 \\
\hline Participant 10 & $\begin{array}{l}\text { Between hatch } \\
\text { and contour }\end{array}$ & 0.12 & 1.44 & 1.0 \\
\hline
\end{tabular}

Abbreviations used: $\mathrm{CAD}=$ computer aided design

\subsection{Individual results}

Participant 2 reported results very similar to the reference (Participant 1 ) in all aspects, as shown in Figure 3. For the cube, the mean porosity was reported as 0.012 percent (reference 0.017 percent) with maximum pore size $0.210 \mathrm{~mm}$ (reference $0.190 \mathrm{~mm}$ ). The cube material volume is reported as $900.6 \mathrm{~mm}^{3}$ (reference $915.8 \mathrm{~mm}^{3}$ ) and a surface roughness colourmap was successfully generated, 
with a mean $S_{a}$ value of -0.00113 (no unit reported) which indicates an incorrect calculation of the $S_{a}$ value, despite using correct image analysis steps (as is clear in Figure 3c). In this case, most likely the positive values were offset by the negative values (all added directly) instead of obtaining an absolute positive variation value as is required in the calculation. The contour porosity in the bracket is clearly identified with a mean porosity 0.04 percent ( 0.056 percent reference) and a maximum pore size of $1.58 \mathrm{~mm}$ ( $0.75 \mathrm{~mm}$ reference). The larger detected pore is in a region of contour porosity which was not detected in the reference bracket scan. Technically, no protocol was provided for this analysis so differences in settings of the defect analysis procedure used in VGSTUDIO MAX will result in different amounts of contour pores detected. The challenge is that pores close to the surface may be seen as part of the surface and hence not included, or beam hardening artefacts near the edges make it difficult to include all contour pores in the analysis. The bracket deviation from CAD is very similar to that of the reference showing the largest warping occurring on the two upright arms towards one another an amount reported as $>0.5 \mathrm{~mm}$ (the actual value was not measured). The layer flaw is clearly shown in the image in Figure 3F. 

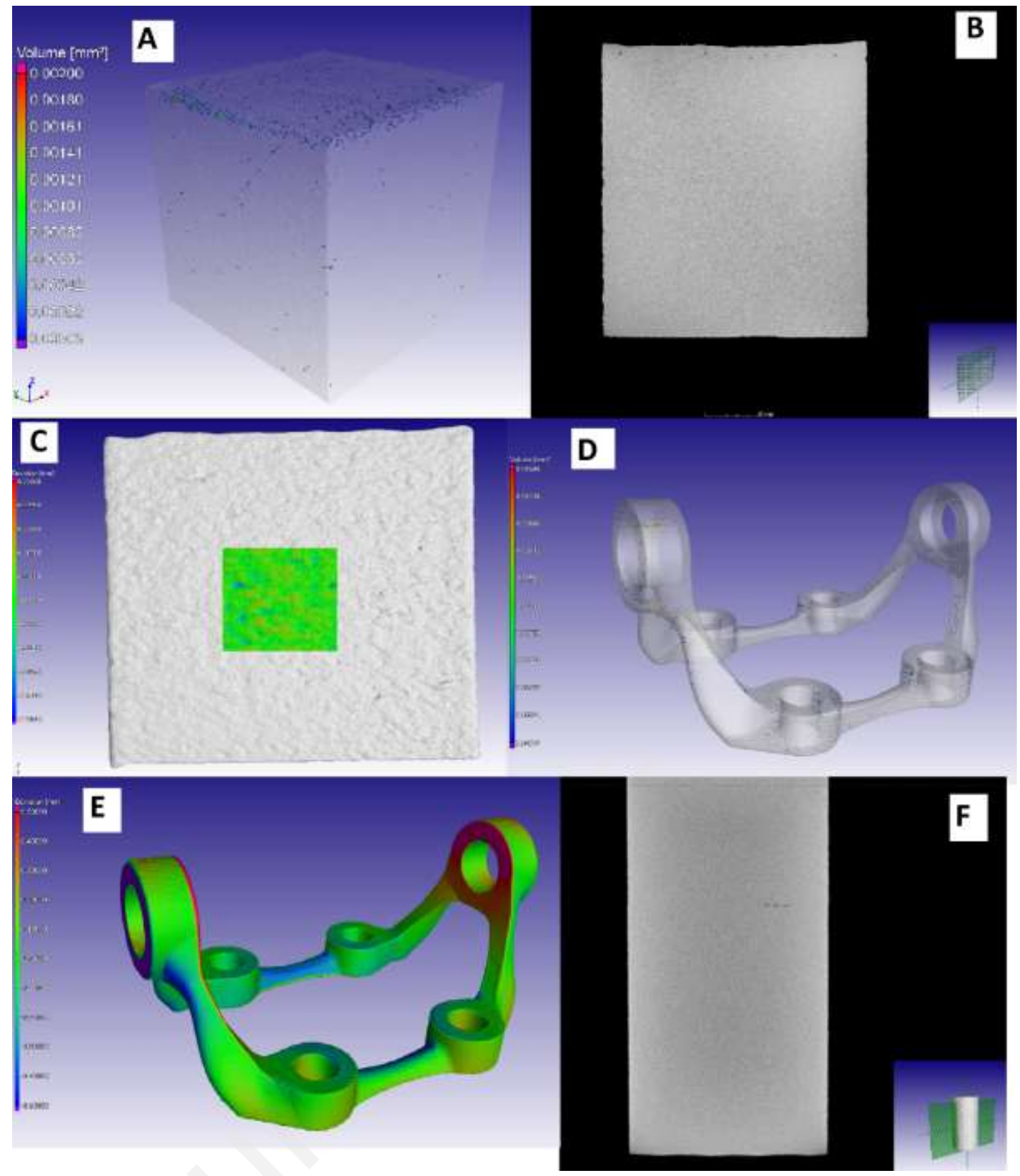

Figure 3: Participant 2 results closely resemble those of the reference: (A) 3D porosity distribution of cube with pores at one surface only (B) 2D porosity slice image of cube (C) surface roughness colourmap (D) bracket 3D porosity analysis showing contour pores around sides of bracket (E) bracket 3D colourmap showing warping and (F) layered flaw in slice image of witness specimen (rod).

Participant 3 reported that a ruby ball bar was used for dimensional calibration prior to the scan, and used additional beam filters $(0.5 \mathrm{~mm}$ copper $+0.5 \mathrm{~mm}$ tin, compared to $0.5 \mathrm{~mm}$ copper only for reference). The porosity value of 0.020 percent is close to the reference ( 0.017 percent) and the images 
also closely resemble the reference as shown in Figure 4, but the largest pore size of $0.285 \mathrm{~mm}$ is larger than found by reference $(0.190 \mathrm{~mm})$. This might be due to two or more small nearest neighboring pores (a pore cluster) being detected as a single pore, which may be caused by small segmentation differences. Total material volume was reported as $841.6 \mathrm{~mm}^{3}$, much lower than the reference and other participants. This might be due to volume measurement of the sub-surface region selected for porosity analysis instead of the entire cube. The surface roughness measurement could not be completed by this participant. The bracket porosity analysis shows contour pores with maximum value of $0.48 \mathrm{~mm}$ (reference $0.75 \mathrm{~mm}$ ), and mean porosity 0.020 percent. The CAD variance is similar to the reference with a maximum deviation of $0.93 \mathrm{~mm}$ reported (reference $1.12 \mathrm{~mm}$ ). The layered flaw is detected but the image provided shows the surface determination (white dots) present, obscuring its clear view.

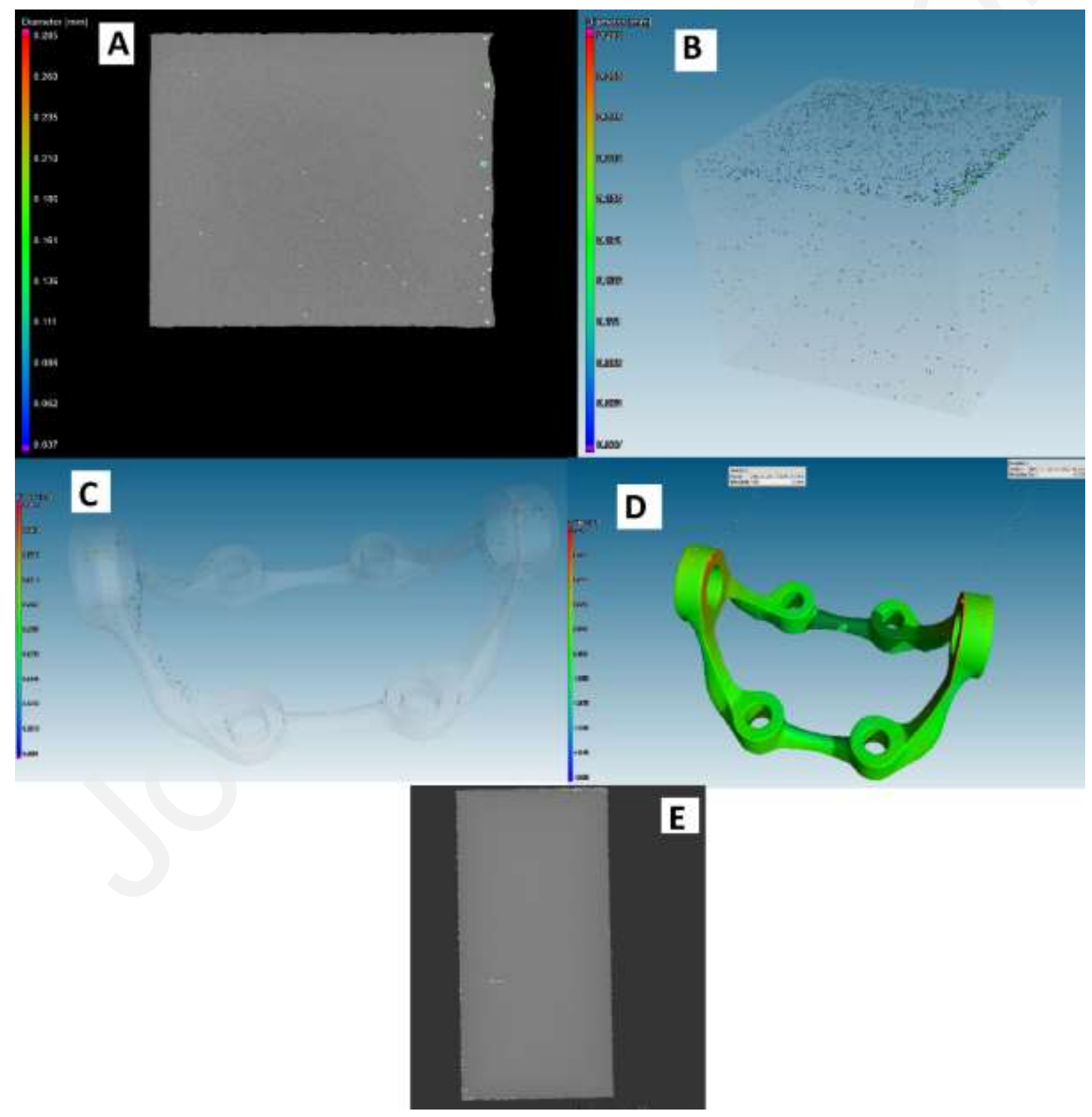


Figure 4: Results of Participant 3, also close to reference: (A) 2D porosity slice image of cube (B) 3D porosity distribution of cube with pores at one surface only (C) bracket 3D porosity analysis showing contour pores around sides of bracket (D) bracket 3D colourmap showing warping and maximum warping annotations (E) layered flaw in slice image of witness specimen (rod).

Participant 4 reported a pore size of $0.54 \mathrm{~mm}$ and a mean porosity of 0.020 percent for the cube, in both cases slightly more than the reference lab. This participant mounted the cube at 45 degrees in one axis instead of 45 degrees in both axes as in the protocol, due to a misreading of the protocol. The loading at 45 degrees in only one axis results in two sides and four edges being subjected to exposures parallel to the X-ray beam, causing slight cone beam artefacts on these edges which make the area under surface less bright in these areas, leading to the possibility of false pore detection. Nevertheless, the porosity distribution is correct and the correct cube was identified. The surface roughness colourplot seems reasonable as shown in Figure $5 C$, with reported value of $4.5 \mu \mathrm{m}$ lower than the reference lab of $8.0 \mu \mathrm{m}$ - this can be explained by the fact that the reference lab measurement was done on a vertical wall, which has a higher roughness than the top surface measured here. The bracket porosity analysis shows in Figure 5D that some large areas below the surface are included in the porosity analysis incorrectly, which explains the higher mean porosity of 0.14 percent and larger pore size than expected of $6.46 \mathrm{~mm}$. This is caused by dark streak artifacts which was not taken in an account within operator's threshold selection. The CAD variance is good with a maximum deviation value of $1.02 \mathrm{~mm}$ (reference $1.12 \mathrm{~mm}$ ). The layered flaw in the rod is clearly visualized in Figure 5F. 


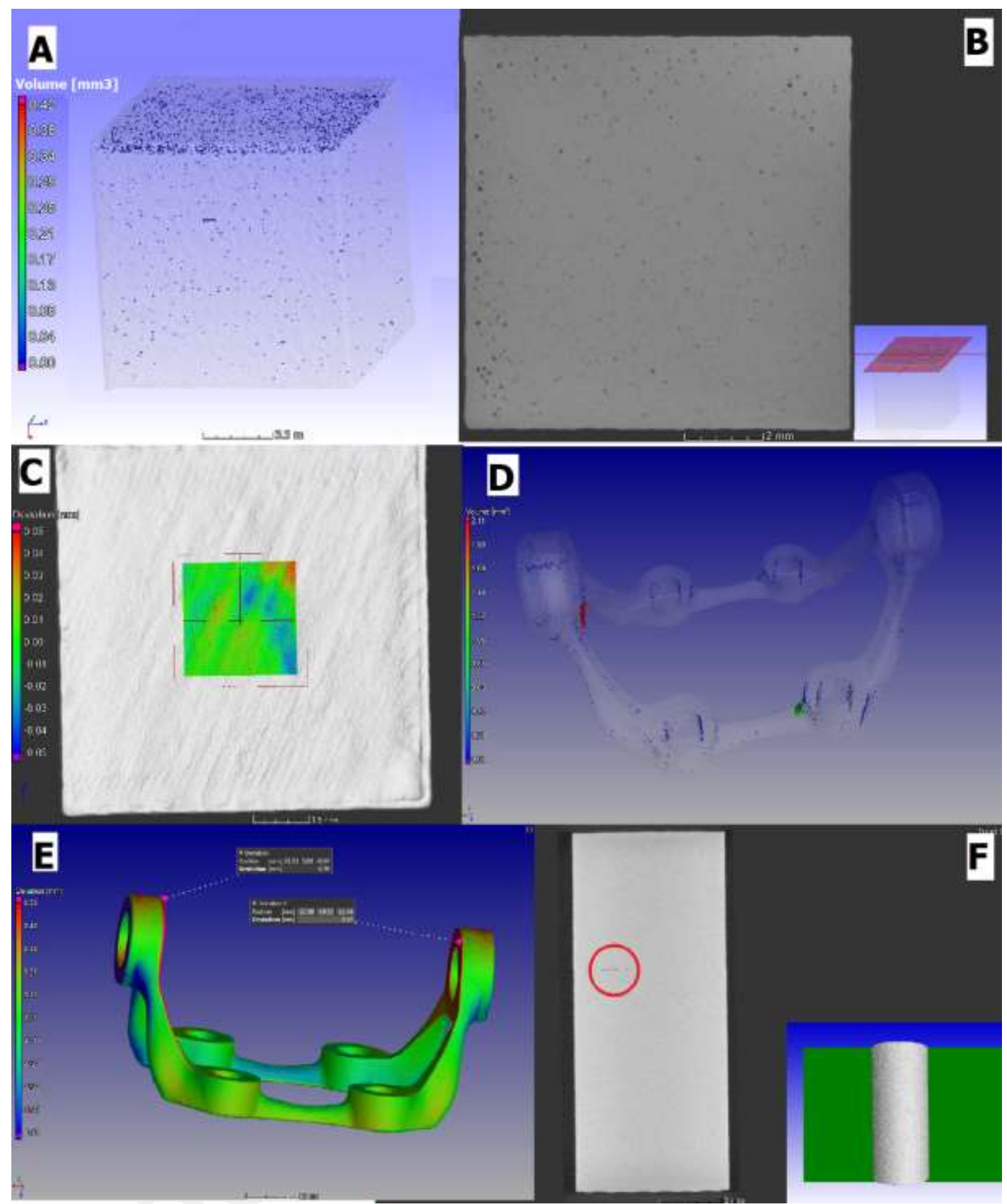

Figure 5: Participant 4 results: (A) 3D porosity distribution of cube with pores at one surface only (B) 2D porosity slice image of cube in plane of pores (C) surface roughness colour-map on top surface (D) bracket 3D porosity analysis showing large false pore detection under surface in various locations $(E)$ bracket 3D colourmap showing warping and maximum deviation annotations (F) layered flaw in slice image of witness specimen (rod). 
Participant 5 reported a $0.85-\mathrm{mm}$ maximum pore size value for the cube which is too large (the mean porosity was also too large at 0.03 percent). When carefully inspecting the images it can be seen that there is an image double-edge (in Figure $6 \mathrm{~A}$ and $\mathrm{D}$ ), which can be caused by incorrect rotation axis position or rotation axis angular alignment (not perpendicular to the X-ray beam). Rotation axis position can be corrected in reconstruction but in this case could not be improved, which indicates rotational axis angular misalignment. This causes pores to seem larger than expected, with a "blurred out" effect visible. It is expected that dimensional measurements are affected by this. Nevertheless, the total cube volume is reported as $931.9 \mathrm{~mm}^{3}$ (reference $915.8 \mathrm{~mm}^{3}$ ), and a surface roughness $\mathrm{S}_{a}$ value could be calculated as $5.4 \mu \mathrm{m}$ (reference $8.0 \mu \mathrm{m}$ ). Due to the double edges present, bracket porosity was not assessed. The CAD variance was reported as $0.85 \mathrm{~mm}$. Despite the image quality problems, the layered flaw in the rod is still visualized clearly. 


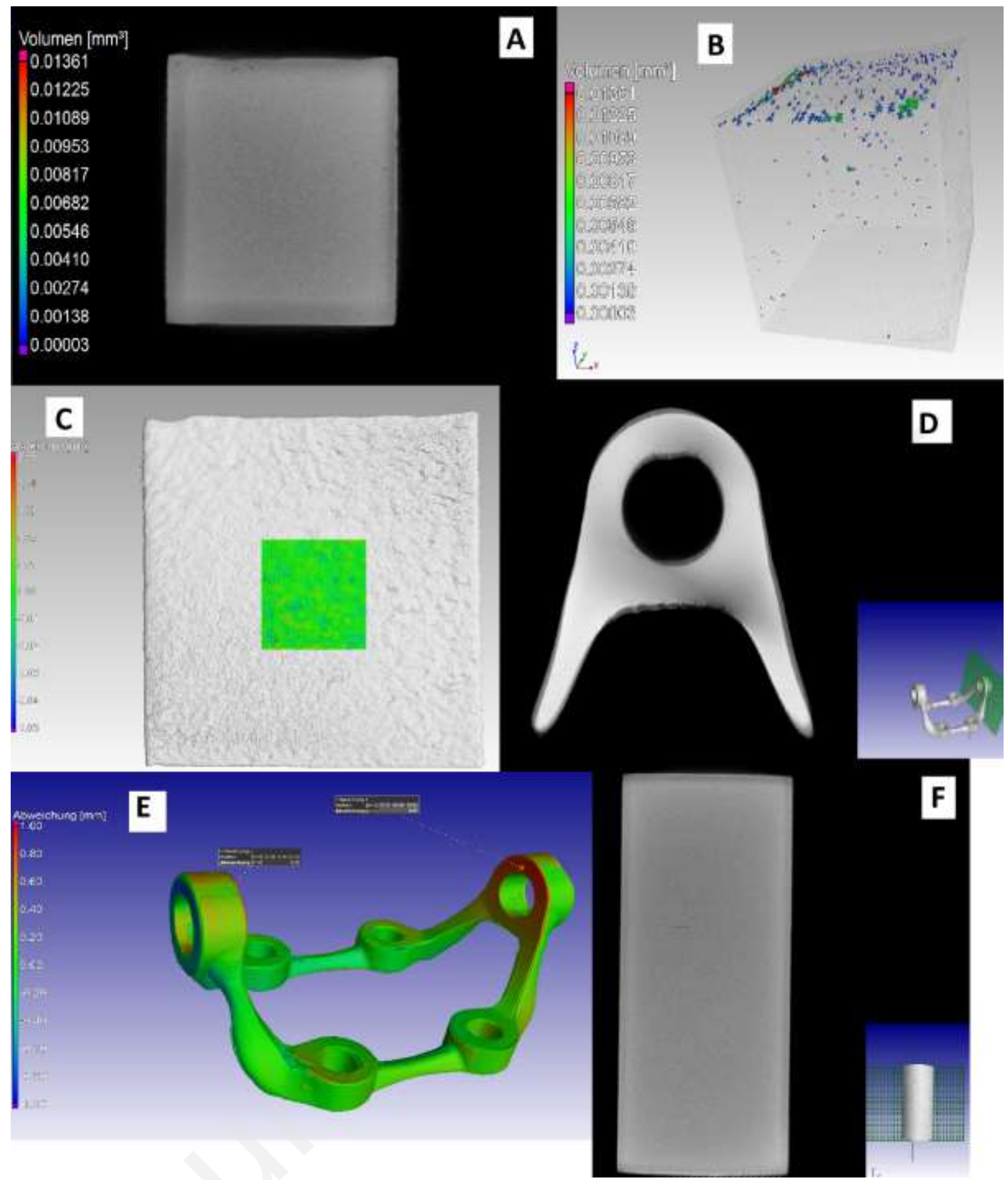

Figure 6: Participant 5 results show double edge present which affects all measurements. (A) 2D porosity slice image of cube (B) 3D porosity distribution of cube with pores at one surface only (C) surface roughness colour-map (D) bracket slice image showing double edge indicating scan error (E) bracket 3D colourmap showing warping and maximum deviation annotations (F) layered flaw in slice image of witness specimen (rod).

Participant 6 reported similar results than the reference for all analyses except the CAD variance as shown in Figure 7. For the cube, the pores are clearly visualized with the correct distribution. The mean 
porosity is reported as 0.020 percent (reference 0.017 percent) and maximum pore size is reported as $0.41 \mathrm{~mm}$, but it is indicated that this was in fact a pore cluster. The surface roughness contour plot is good, but an $\mathrm{S}_{\mathrm{a}}$ value could not be calculated. For the bracket the largest pore is reported as $0.76 \mathrm{~mm}$ (reference $0.75 \mathrm{~mm}$ ). However, the CAD variance analysis was an issue - clearly the alignment was not ideal and this process needs some refinement in the protocol.

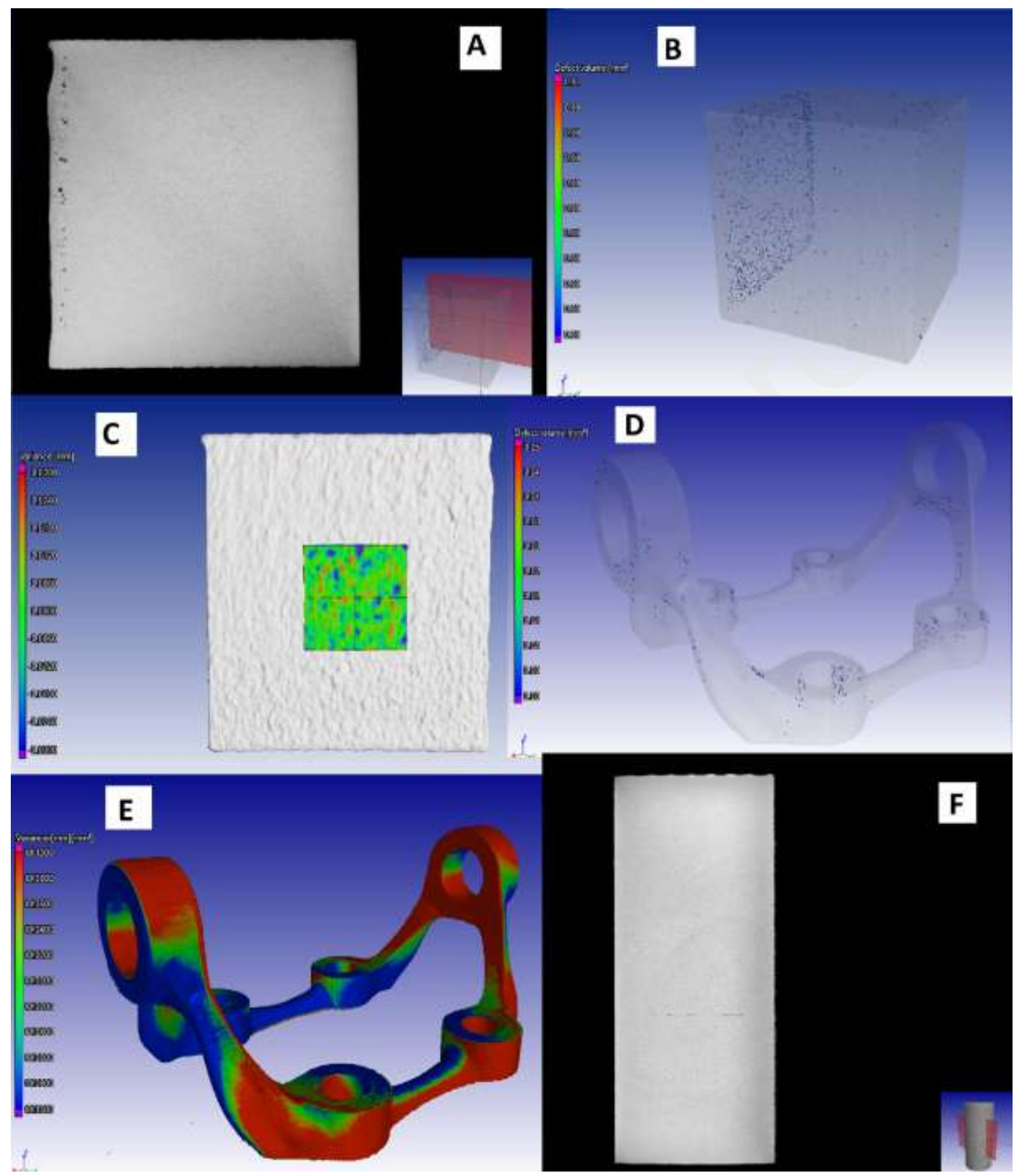

Figure 7: Participant 6 results all correspond to reference except the CAD variance: (A) 2D porosity slice image of cube (B) 3D porosity distribution of cube with pores at one surface only (C) surface roughness colour-map (D) bracket 3D porosity analysis showing contour pores around sides of bracket (E) bracket 
3D colourmap showing large variations incorrectly due to incorrect overlap of CAD file with actual data (F) layered flaw in slice image of witness specimen (rod).

Participant 7 (Figure 8) submitted results in two formats: one following the protocol exactly and one an improved modified version - the improved data is reported here only. Both CT scanning parameters and subsequent data analysis via VGSTUDIO MAX 3.2 have been slightly adjusted to avoid CT artefacts. For scanning parameters, exposure time has been increased while total scanning power has been decreased to reach the minimum X-Ray gun spot size and therefore increase spatial resolution. Regarding data analysis, surface determination for the cube [19] was performed by the participant with "advanced surface determination, removing all particles and voids" in VGSTUDIO, due to some inherited noise caused by sample positioning.

Porosity of the cube is reported as 0.018 percent (reference 0.017 percent), with maximum pore size $0.26 \mathrm{~mm}$ (reference $0.19 \mathrm{~mm}$ ). Surface roughness was calculated as $S_{a}=5.4 \mu \mathrm{m}$ (reference $8.0 \mu \mathrm{m}$ ). The lower value noted for $S_{a}$ relative to the reference value could be due to different areas selected for analysis, as only a part of the surface is analyzed. The total cube volume is measured as $904.3 \mathrm{~mm}^{3}$ (reference $915 \mathrm{~mm}^{3}$ ). The bracket analysis shows contour porosity with largest pore size $0.61 \mathrm{~mm}$ (reference $0.75 \mathrm{~mm}$ ) and CAD variance analysis shows maximum warping of $0.94 \mathrm{~mm}$ (reference 1.12 $\mathrm{mm})$. The results from this participant were not as good when following the protocol exactly. For example, two large pores on two opposing surfaces of the cube were identified incorrectly as pores but were in fact due to segmentation error. This could be caused, in turn, by the loading of the sample at 45 degrees in one axis only, similar to what was noted for Participant 4. As reported, surface determination was performed with an "advanced surface determination removing all particles and voids" which apparently results in less chance of artefact areas being included. Nevertheless, the use of the simpler surface determination compared to the suggested protocol [19], may lead to in the inclusion of exterior particles - such as shown in Figure $8 \mathrm{C}$ in pink color, which might affect the subsequent surface roughness assessment. It was also highlighted by this participant that the selection of the final ISO threshold value (manual step in the protocol [19]) is as well a critical step, and can result in serious errors. This can currently only be mitigated by manually inspecting the selection in slice images to ensure no false porosity detection. 


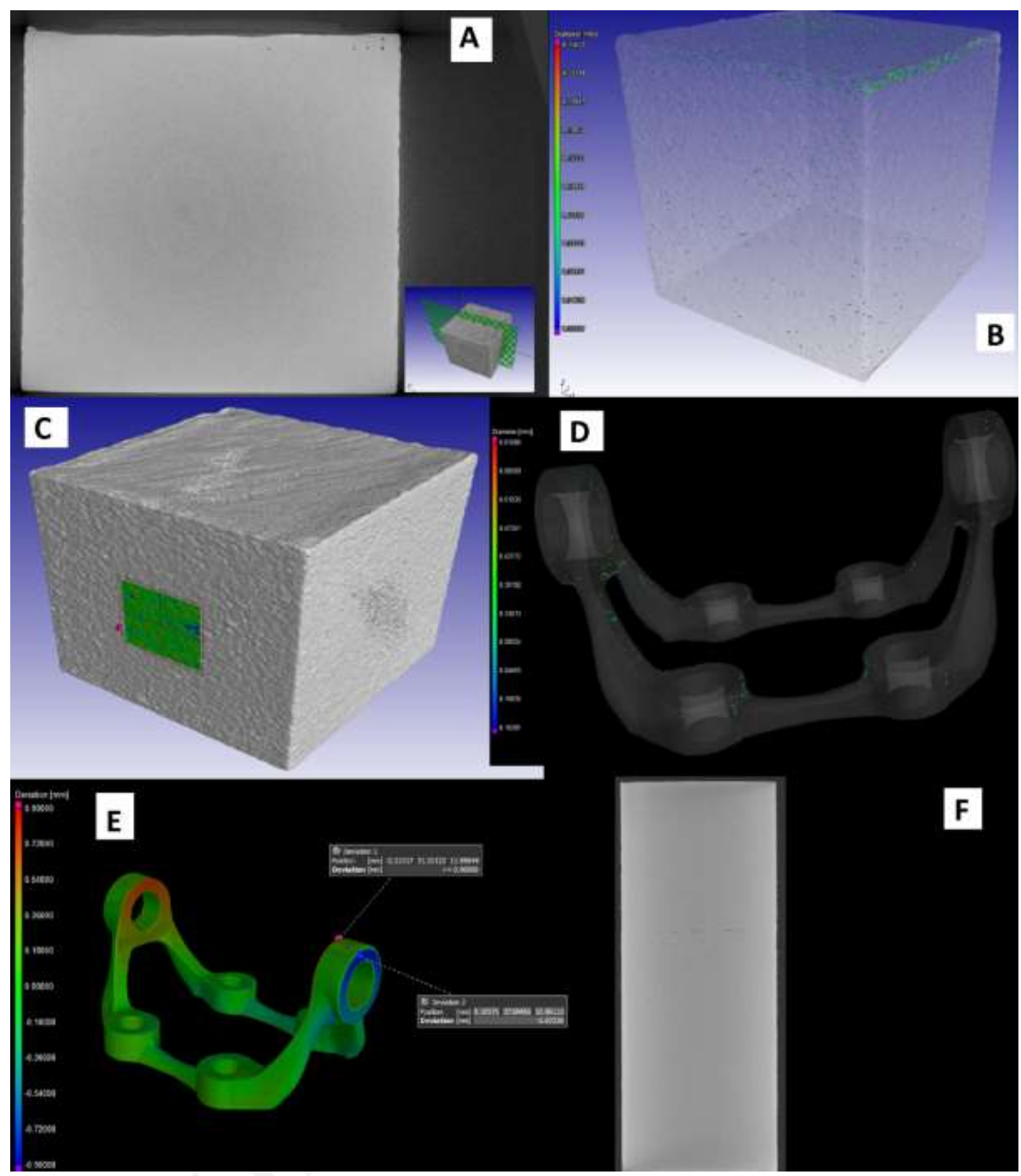

Figure 8: Participant 7 results are similar than those of the reference and was implemented with some modifications to the protocols: (A) 2D porosity slice image of cube (B) 3D porosity distribution of cube with pores at one surface only (C) surface roughness colour-map including as "pink" particle above surface (D) bracket 3D porosity analysis showing contour pores around sides of bracket (E) bracket 3D colourmap showing warping and maximum warping annotations ( $F$ ) layered flaw in slice image of witness specimen (rod). 
Participant 8 reported positive correlation of samples to those in the prior study (pore distribution type). This participant noted that the cube was scanned with no angle (zero degrees = top and bottom cube edges parallel to the X-ray beam and perpendicular to the rotation axis) - leaving edge cone beam artefacts making quantitative analysis impossible. Despite these artefacts, by eliminating falsely detected pores from the analysis (by visual inspection), the mean porosity and the pore size is very near those of the reference. For the bracket, the porosity mean value of 0.060 percent also corresponds well with reference of 0.056 percent. The layered flaw is also clearly identified.

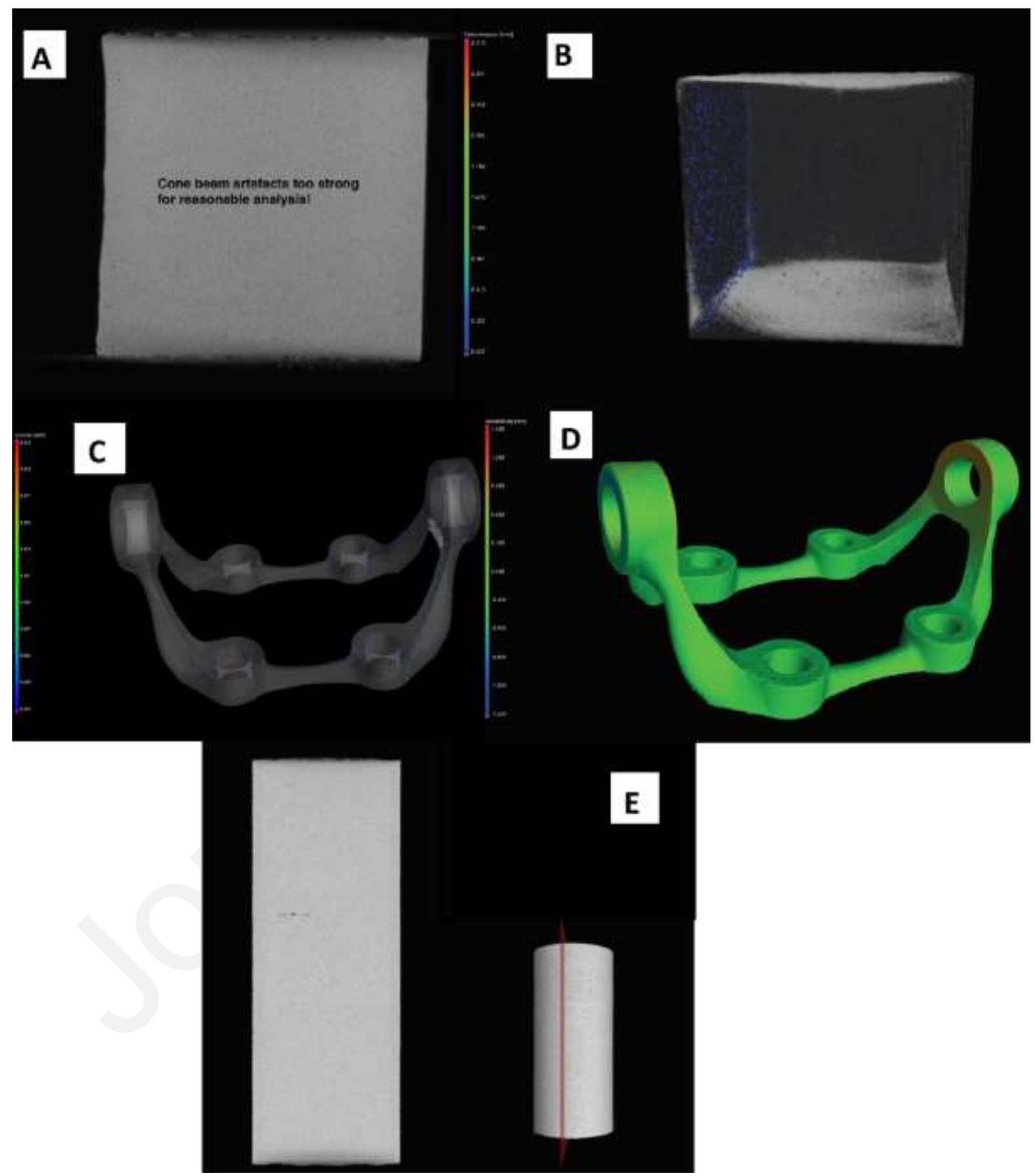

Figure 9: Results of Participant 8 in line with others despite lack of quantitative assessments. (A) 2D porosity slice image of cube (B) 3D porosity distribution of cube with pores at one surface only (C) 
bracket 3D porosity analysis showing contour pores around sides of bracket (D) bracket 3D colourmap showing warping (E) layered flaw in slice image of witness specimen (rod).

Participant 9 (Figure 10) shows reasonable results with the exception of lower mean porosity value of the cube ( 0.004 percent compared to reference 0.017 percent) and clearly less pores identified in the images in Figure 10A and B, compared to other participants. This might be due to lack of contrast due to (possibly) a large source-to-detector distance resulting in noisy data. In this case a modification might be to increase the source brightness, move the detector closer to the source, or apply post-scan image de-noising filters to detect the small pores that were missed without filtering. Nevertheless, the main porosity distribution is clear and the cube total volume is $903.6 \mathrm{~mm}^{3}$, which is close to the reference value. The bracket analysis shows a mean porosity value of 0.067 percent and maximum pore size $0.845 \mathrm{~mm}$. Maximum deviation from CAD is $0.88 \mathrm{~mm}$, which is in line with other participants. 


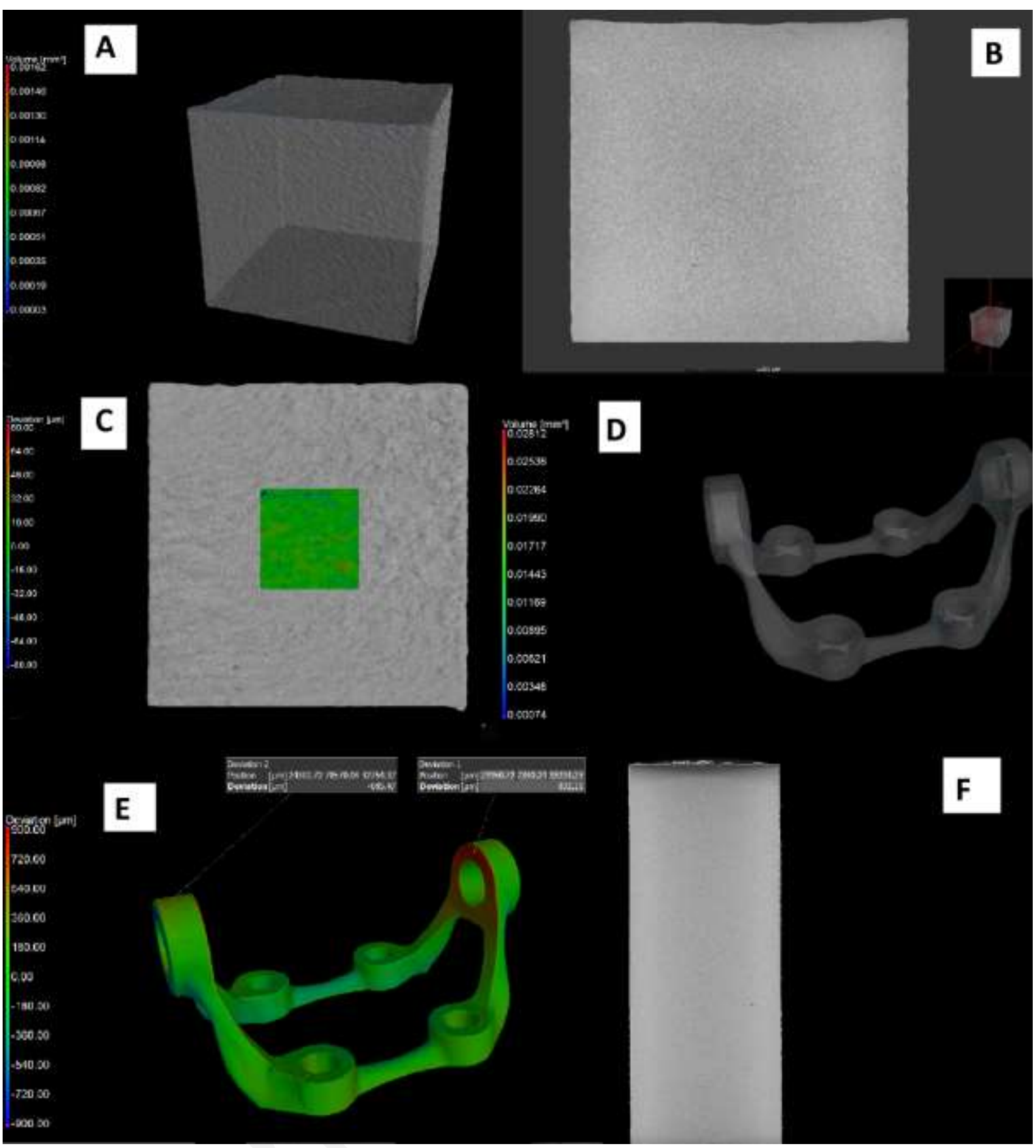

Figure 10: Participant 9 results show reduced contrast but all critical features are identified positively (A) 3D porosity distribution of cube with pores at one surface only (B) 2D porosity slice image of cube (C) surface roughness colour-map (D) bracket 3D porosity analysis showing contour pores around sides of bracket $(E)$ bracket 3D colourmap showing warping and maximum warping annotations $(F)$ layered flaw in slice image of witness specimen (rod).

Participant 10 also scanned the cube at a zero degree angle (without any angle), resulting in artefacts along the cube edges. Due to this artefact area, this participant used another process to determine porosity, namely the VGSTUDIO MAX EasyPore algorithm. Despite the different workflow, the mean 
porosity 0.030 percent and maximum pore size of $0.22 \mathrm{~mm}$ correspond well to reference values, and images clearly show results very similar to the reference. The cube volume was also reported at 941.3 $\mathrm{mm}^{3}$, which is also close to the reference value. Roughness was assessed, giving a value for $S_{a}=4.6 \mu \mathrm{m}$. For the bracket this participant showed contour porosity as expected and CAD deviation value of 1.00 $\mathrm{mm}$ (reference $1.12 \mathrm{~mm}$ ). Again, the layered flaw was positively identified.

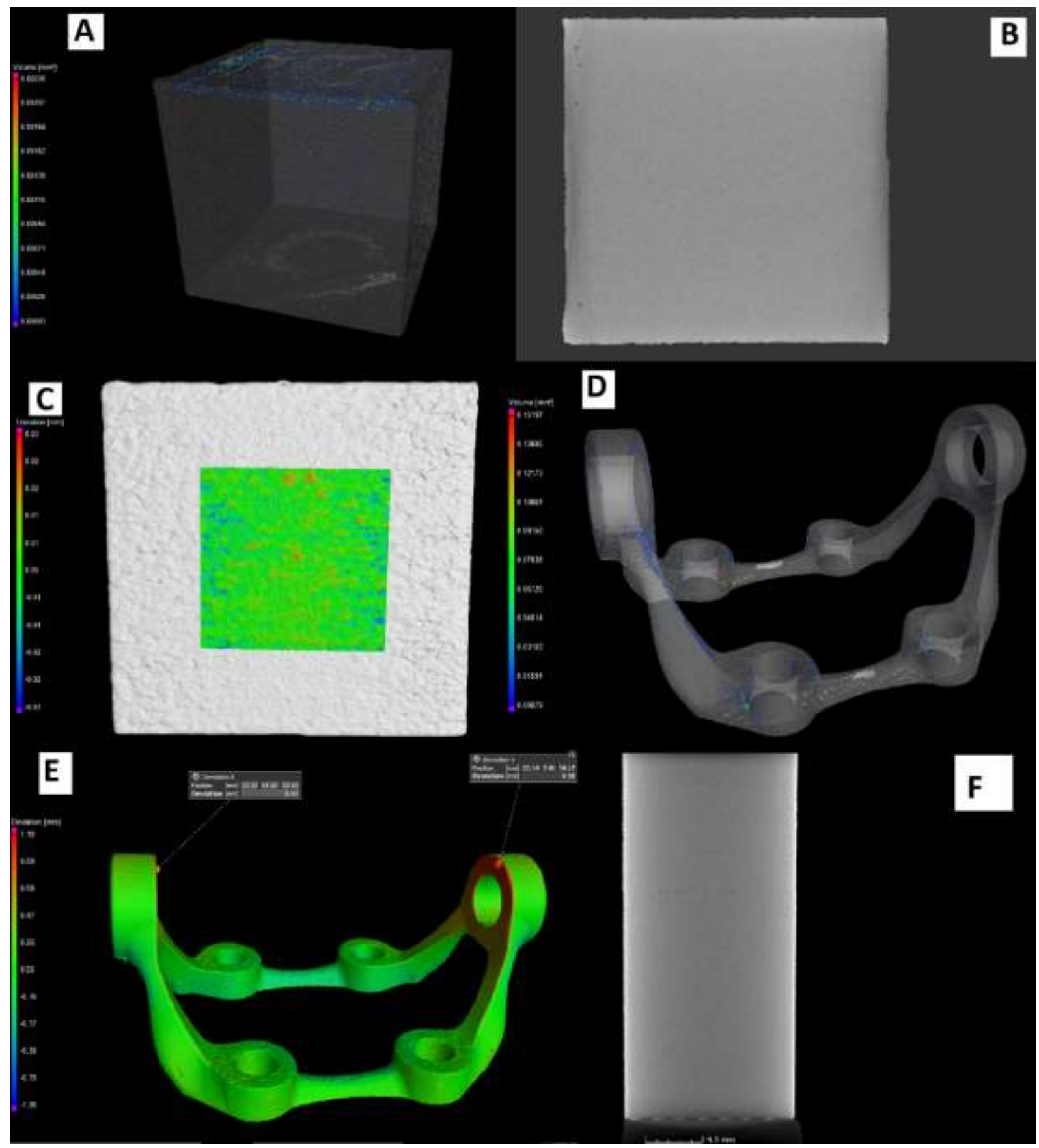

Figure 11: Participant 10 results are in good agreement with others (A) 3D porosity distribution of cube with pores at one surface only (B) 2D porosity slice image of cube (C) surface roughness colour-map (D) bracket 3D porosity analysis showing contour pores around sides of bracket (E) bracket 3D 
colourmap showing warping and maximum warping annotations $(F)$ layered flaw in slice image of witness specimen (rod).

\subsection{Suggested improvements and developments}

It is noteworthy that two participants loaded the cube at zero angle and two others loaded the cube at 45 degrees in one axis only. In both cases, the flat surfaces are exposed to projections parallel to the $X$-ray beam, resulting in cone beam artefacts, which can not only affect quantitative assessment of porosity, but also can hide critical flaws. The only way to overcome this is to ensure no flat surfaces are loaded parallel to the beam direction. Luckily, additive manufactured parts are typically complex in shape and lack flat surfaces. For the protocol, the cube must simply be loaded at a 45 degree angle in two axes. An extremely important point here is the fact that lack of fusion and layered flaws occur in the build plane. Therefore, for best image quality and to reduce the chance of artefacts hiding these very narrow and flat flaws, AM parts must be scanned at an angle relative to the build plane (which is usually known or easy to see on the as-built surface).

The selection of the surface of the cube (surface determination) was discussed in the context of Participant 7, who showed that a simpler method is available. However, this method can include exterior particles. Future software developments might therefore assist in this effort to ensure no exterior particles or interior cavities are included in this step. This participant also highlighted the fact that, despite the protocol's removal of exterior air, the thresholding step is still a manual process with potential error. It is expected that as the porosity gets smaller in size and quantity this error becomes larger. The only way to mitigate this at present is to check all slices manually to ensure the selection does not include noise or miss pores. It should be mentioned that the local optimization used in the software tool does remove much of the human threshold error, but it requires a good threshold value to start with.

The results show that contrast and image quality differs among participants - despite the availability of a protocol, each system is different and some settings cannot be reproduced exactly. The most important criteria for successful analysis is to ensure that the image contrast is good enough so that all critical flaws are detected. While all participants positively identified all critical flaws and porosity distributions, it is clear from the images that the background noise and image contrast (image quality) varies. There is no currently accepted method to directly compare image quality and this is something that requires future development. In the meantime, some suggested improvements to the protocols include: use longer exposures to improve contrast, move the detector closer to the source if possible, and to use frame averaging to reduce noise if possible. It might also be possible to reduce noise using 
image filtering options, which was not implemented in this work and might simplify the workflow considerably.

Since the surface roughness measurement could not be completed by some participants, these steps need to be described more clearly in the protocols. The alignment of the CAD file with the actual geometry also requires better description.

\section{Conclusions}

A round robin test was conducted using recently proposed standard micro-CT scan and image analysis protocols, for representative coupon samples. The main result is that the protocols work for qualitative assessment and identification of flaws and characteristic porosity distributions (porosity distribution in cube and bracket, warping in bracket, layer flaw in witness rod) but deviations are found in all quantitative assessments despite the protocols used.

Sources of deviation were discussed and these could be categorized either as scanning errors or image analysis errors. Scanning errors can be prevented by more detailed instructions, for example, clearly specifying the scan orientation of sample relative to the X-ray beam. To mitigate image analysis errors, future work should involve development of modifications for each particular commercial micro-CT system type as all micro-CT systems have different capabilities which affect image quality in terms of artefacts, background noise levels, contrast between background and material, instrument detail detectability and edge sharpness. Alternatively, image quality metrics need to be developed to ensure sufficient image quality is available for the required analysis. Image analysis is challenging and for small porosity always will result in slight differences in results. This highlights the need for even more strict guidelines on image analysis with included (manual) checkpoints to ensure analysis is performed accurately despite some differences in image quality. One potential route to assist with this is to provide the reference data obtained (Participant 1 cube) in its raw format and allow readers to perform their own analysis on this data, which is provided as supplementary material. Especially during the segmentation step in the porosity analysis of the cube (when creating the defect mask for analysis), the potential human error can be reduced by training and experience. This can be supported by additional images in the protocols, highlighting correct and incorrect selections and what to check for. The implementation of X-ray micro-CT inspections for coupon samples for process optimization hold great potential for improving AM processes and parts. 


\section{Acknowledgements}

The South African Department of Science and Technology is acknowledged for support through the Collaborative Program for Additive Manufacturing (CPAM).

This research was carried out under the project CEITEC 2020 (LQ1601) with financial support from the Ministry of Education, Youth and Sports of the Czech Republic under the National Sustainability Programme II and support of CEITEC Nano Research Infrastructure (MEYS CR, 2016-2019).

Mirko Sinico kindly acknowledges the funding from the H2020-MSCA-ITN-2016 project PAM ${ }^{2}$ (Precision Additive Metal Manufacturing), EU Framework Programme for Research and Innovation H2020 Grant Agreement No 721383. 


\section{References}

[1] T. Debroy, H.L. Wei, J.S. Zuback, T. Mukherjee, J.W. Elmer, J.O. Milewski, A.M. Beese, A. Wilson-Heid, A. De, W. Zhang, Additive manufacturing of metallic components - Process, structure and properties, Prog. Mater. Sci. 92 (2018) 112-224. doi:10.1016/j.pmatsci.2017.10.001.

[2] D. Bourell, J.P. Kruth, M. Leu, G. Levy, D. Rosen, A.M. Beese, A. Clare, Materials for additive manufacturing, CIRP Ann. 66 (2017) 659-681. doi:10.1016/J.CIRP.2017.05.009.

[3] D.D. Gu, W. Meiners, K. Wissenbach, R. Poprawe, Laser additive manufacturing of metallic components: materials, processes and mechanisms, Int. Mater. Rev. 57 (2012) 133-164. doi:10.1179/1743280411Y.0000000014.

[4] A. du Plessis, C. Broeckhoven, I. Yadroitsava, I. Yadroitsev, C.H. Hands, R. Kunju, D. Bhate, Beautiful and Functional: A Review of Biomimetic Design in Additive Manufacturing, Addit. Manuf. (2019). doi:10.1016/J.ADDMA.2019.03.033.

[5] E. Todorov, R. Spencer, S. Gleeson, M. Jamshidinia, S.M. Kelly, 2014 America Makes NDE of metallic structures interim report, (2014).

[6] B. Dutton, W. Vesga, J. Waller, S. James, M. Seifi, Metal Additive Manufacturing Defect Formation and Non-Destructive Evaluation (NDE) Detectability, in: M. Seifi, N. Shamsaei (Eds.), ASTM STP 1620, 2018.

[7] J. Damon, S. Dietrich, F. Vollert, J. Gibmeier, V. Schulze, Process dependent porosity and the influence of shot peening on porosity morphology regarding selective laser melted AISi10Mg parts, Addit. Manuf. 20 (2018) 77-89. doi:10.1016/j.addma.2018.01.001.

[8] A.D. Brandão, J. Gumpinger, M. Gschweitl, C. Seyfert, P. Hofbauer, T. Ghidini, Fatigue Properties of Additively Manufactured AlSi10Mg-Surface Treatment Effect, Procedia Struct. Integr. 7 (2017) 58-66. doi:10.1016/j.prostr.2017.11.061.

[9] W. Dewulf, M. Pavan, T. Craeghs, J.P. Kruth, Using X-ray computed tomography to improve the porosity level of polyamide-12 laser sintered parts, CIRP Ann. - Manuf. Technol. 65 (2016) 205-208. doi:10.1016/j.cirp.2016.04.056.

[10] A. du Plessis, I. Yadroitsev, I. Yadroitsava, S.G. Le Roux, X-Ray Microcomputed Tomography in Additive Manufacturing: A Review of the Current Technology and Applications, 3D Print. Addit. Manuf. 5 (2018) 3dp.2018.0060. doi:10.1089/3dp.2018.0060. 
[11] M. Seifi, A. Salem, J. Beuth, O. Harrysson, J.J. Lewandowski, Overview of Materials Qualification Needs for Metal Additive Manufacturing, JOM. 68 (2016) 747-764. doi:10.1007/s11837-015-1810-0.

[12] ASTM E1570-11, Standard Practice for Computed Tomographic (CT) Examination, ASTM International, West Conshohocken, PA, (2011).

[13] ASTM WK47031, Standard Guide for Nondestructive Testing of Metal Additively Manufactured Aerospace Parts After Build, Draft standard, ASTM International, West Conshohocken, PA, (2019). https://www.astm.org/Workltems/WK47031.htm, last accessed May 23, 2019.

[14] ISO/ASTM DTR 52905 (ASTM F42) Additive manufacturing -- General principles -- Nondestructive testing of additive manufactured products, under development, ISO Technical Committee 261 (2019)., 2019. https://www.iso.org/standard/71988.html, last accessed May 23, 2019.

[15] W. Dewulf, M. Ferrucci, E. Ametova, P. Heřmánek, G. Probst, B. Boeckmans, T. Craeghs, S. Carmignato, Enhanced dimensional measurement by fast determination and compensation of geometrical misalignments of X-ray computed tomography instruments, CIRP Ann. 67 (2018) 523-526. doi:10.1016/j.cirp.2018.04.124.

[16] M. Ferrucci, R.K. Leach, C. Giusca, S. Carmignato, W. Dewulf, Towards geometrical calibration of x-ray computed tomography systems - A review, Meas. Sci. Technol. 26 (2015). doi:10.1088/0957-0233/26/9/092003.

[17] P. Hermanek, F. Zanini, S. Carmignato, Traceable Porosity Measurements in Industrial Components Using X-Ray Computed Tomography, J. Manuf. Sci. Eng. 141 (2019) 051004. doi:10.1115/1.4043192.

[18] F.H. Kim, S.P. Moylan, E.J. Garboczi, J.A. Slotwinski, Investigation of pore structure in cobalt chrome additively manufactured parts using X-ray computed tomography and threedimensional image analysis, Addit. Manuf. 17 (2017) 23-38. doi:10.1016/J.ADDMA.2017.06.011.

[19] A. du Plessis, P. Sperling, A. Beerlink, L. Tshabalala, S. Hoosain, N. Mathe, G. Stephan, MethodsX Standard method for microCT-based additive manufacturing quality control 1 : Porosity analysis, MethodsX. 5 (2018) 1102-1110. doi:10.1016/j.mex.2018.09.005.

[20] A. du Plessis, P. Sperling, A. Beerlink, L. Tshabalala, S. Hoosain, N. Mathe, G. Stephan, MethodsX Standard method for microCT-based additive manufacturing quality control 2 : 
Density measurement, MethodsX. 5 (2018) 1117-1123. doi:10.1016/j.mex.2018.09.006.

[21] A. du Plessis, P. Sperling, A. Beerlink, O. Kruger, L. Tshabalala, S. Hoosain, S.G. le Roux, Standard method for microCT-based additive manufacturing quality control 3: Surface roughness, MethodsX. 5 (2018) 1111-1116. doi:10.1016/j.mex.2018.09.006.

[22] A. du Plessis, P. Sperling, A. Beerlink, W. du Preez, S.G. le Roux, Standard method for microCTbased additive manufacturing quality control 4: Metal powder analysis, MethodsX. 5 (2018) 1336-1345. doi:10.1016/j.mex.2018.09.006.

[23] https://www.protocols.io/ A collaborative platform and preprint server for science methods, computational workflows, clinical trials, operational procedures, safety checklists, and instructions / manuals, n.d. https://www.protocols.io/ (accessed May 23, 2019).

[24] A. du Plessis, S.G. le Roux, Standardized X-ray tomography testing of additively manufactured parts: a round robin test, Addit. Manuf. (2018). doi:10.1016/J.ADDMA.2018.09.014. 